\title{
Controlling Planar and Vertical Ordering in Three-Dimensional (In,Ga)As Quantum Dot Lattices by GaAs Surface Orientation
}

\author{
M. Schmidbauer ${ }^{1}$, Sh. Seydmohamadi ${ }^{2}$, D. Grigoriev ${ }^{3}$, Zh. M. Wang ${ }^{2}$, Yu. I. Mazur ${ }^{2}$, \\ P. Schäfer ${ }^{3}$, M. Hanke ${ }^{4}$, R. Köhler ${ }^{3}$, and G. J. Salamo ${ }^{2}$ \\ ${ }^{1)}$ Institut für Kristallzüchtung, D-12489 Berlin, Germany \\ ${ }^{2)}$ Department of Physics, University of Arkansas, Fayetteville, Arkansas, 72701, USA \\ ${ }^{3)}$ Institut für Physik, Humboldt-Universität zu Berlin, D-12489 Berlin, Germany \\ ${ }^{4)}$ Martin-Luther-Universität Halle-Wittenberg, D-06120 Halle/Saale, Germany \\ (Submitted 25.08.2005, revised manuscript received 30.11.2005)
}

\begin{abstract}
Anisotropic surface diffusion and strain are used to explain the formation of three-dimensional (In,Ga)As quantum dot lattices. The diffusion characteristics of the surface coupled with the elastic anisotropy of the matrix, provides an excellent opportunity to influence the dot positions. In particular, quantum dots that are laterally organized into long chains or chessboard two-dimensional arrays vertically organized with strict vertical ordering or vertical ordering that is inclined to the sample surface normal are accurately predicted and observed.
\end{abstract}

PACS Numbers: 68.65.-k, 68.35.Fx, 61.10.-i,81.15.Hi

During the last decade semiconductor quantum dots (QDs) have attracted increasing attention because of potential applications as novel semiconductor devices [1,2]. Besides time consuming techniques using electron beam lithography and subsequent etching to fabricate QDs, selforganized growth techniques have captured research interest[2-6]. In the Stranski-Krastanow growth mode, while the growth conditions can be optimized to produce nanostructures of near identical size and shape, often only a random spatial distribution of the QD is observed for a single layer of QDs [7]. However, for multiple layers a range of different results, from near perfect QD chains to threedimensional (3D) lattices, have been reported and discussed [8-11]. In this case, it has been suggested that the anisotropy in surface diffusion for (In,Ga)As QDs on GaAs (100), which is mainly caused by the $(2 \times 4)$ surface reconstruction with dimer rows running along [0-11], is responsible for the formation of QD chains along the [0-11]-direction [9,12]. In particular, the surface diffusion length along [0-11] is larger than along [011]. This leads to greater strain relaxation along the [0-11]-direction, producing an elliptical strain relief that is transferred to succeeding layers. This, eventually causes an asymmetric separation between neighboring dots and consequently leads to QD chain structures. Another example of the outcome of multiple layers of QD growth is the $\mathrm{PbSe} / \mathrm{PbEuTe}$ system where a nearly perfect $3 \mathrm{D}$ lattice is reported along with the suggestion that the self-organized result is caused by anisotropic strain transfer from QD layerto-QD layer [10,11]. In each case the explanation is qualitative and a quantitative understanding of the role of diffusion and strain and the corresponding ability to design 3D QD structures is still lacking.

In this letter, we report on experiments that use natural surface steps on high index substrates to further uncover the role of both surface diffusion and strain in producing 3D ordering of QDs. In particular we examine the formation and development of 3D square-like lattices of (In,Ga)As QDs in a GaAs matrix. The square lattices are created by vertically stacking QD layers while simultaneously introducing surface steps in each layer in order to vary and control the symmetry of the diffusion and strain pattern in each layer. Our findings show that by using different high index substrates we can in fact use surface steps to fine tune surface diffusion and strain in order to encourage 3D organized growth that can take the form ranging from a chain-like pattern to a square-like lattice of QDs. For example, for multi-layered growth on the (100) surface of GaAs the 3D array of QDs is laterally aligned as a dot chain while vertically aligned directly along the [100]direction $[9,13,14]$. This situation is dramatically changed, however, when the substrate surface orientation is different from (100). Indeed, for a (311) substrate orientation the 3D array is laterally aligned in a square-like lattice while (as predicted [15]) the vertical alignment of the 3D QDs forms an inclination angle of $\alpha=10^{\circ}$ to the surface normal, towards the [2-3-3]-direction. In fact, our observations show that $\alpha$ varies substantially when the surface orientation is systematically changed from (100) in the direction towards (111)B and is in good agreement with theoretical simulations based on linear elasticity theory.

In order to probe the $3 \mathrm{D}$ ordering of $\mathrm{QD}$ lattices $\mathrm{x}$-ray diffuse scattering has been monitored three-dimensionally by a special multi-detection technique using a CCD-detector [16]. The experiments were carried out using highly brilliant synchrotron radiation from beamlines $\mathrm{W} 1$ and $\mathrm{BW} 2$ at HASYLAB/DESY at a wavelength of $1.55 \AA$. The surfaces under investigation are GaAs (100) and GaAs (n11)B, where $\mathrm{n}$ is equal to $9,7,5,4$ and 3 . The high index surfaces are tilted towards (111)B surface which forms B-type steps running along [011]-direction, while the direction perpendicular to the step edges is [2n-n].

The samples were grown by solid-source molecular beam epitaxy (MBE) on singular (100), and GaAs (n11)B substrates. The surface miscut from the nominal crystallographic orientation has been determined to be smaller than $0.05^{\circ}$. All of the substrates were indium soldered side by side on a molybdenum block. After a half 
micrometer thick GaAs buffer layer grown at $580^{\circ} \mathrm{C}$, the substrate temperature was lowered to $540^{\circ} \mathrm{C}$ for the deposition of the (In,Ga)As/GaAs multi-layered structure. The multilayer structure consists of 16.5 periods of 10 monolayers (ML) $\mathrm{In}_{0.40} \mathrm{Ga}_{0.60} \mathrm{As}$ QDs and $120 \mathrm{ML}$ GaAs spacers. The last layer of QDs was left exposed for topographic atomic force microscopy (AFM) imaging under ambient conditions.

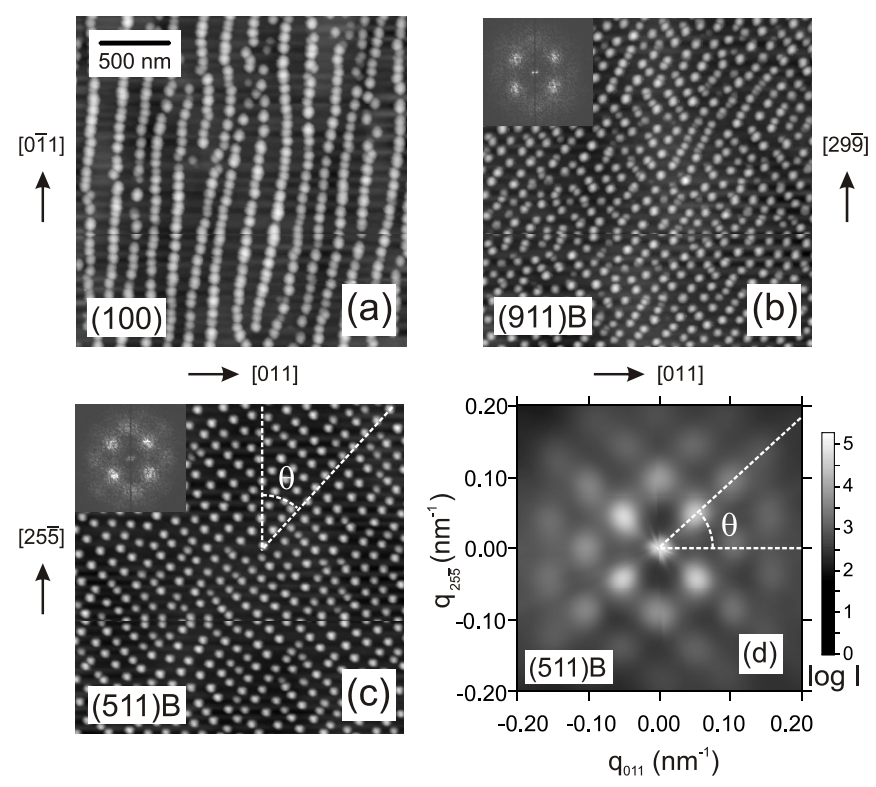

FIG. 1. AFM images of the surface morphology of multi-layered $\mathrm{In}_{0.4} \mathrm{Ga}_{0.6}$ As dots grown on GaAs (100) (a), 511B (b) and (911)B (c) substrates. The insets are fast Fourier transforms taken from the AFM image. The in-plane deflection angle $\theta$ between the nearestneighbor direction and [2n-n]-direction is indicated. (d) Grazingincidence small-angle $\mathrm{x}$-ray scattering.

Our observations of 3D self-organized arrays on a (100) GaAs substrate indicate that the lateral alignment consists of QD chains oriented along the [0-11] direction (Fig. 1(a)) while vertically aligned perpendicular to the (100) surface. This result is consistent with previous work [9]. For the high index (n11)B surfaces under study, B-type surface steps with variable separations are introduced along the [011] direction (perpendicular to the dot chain direction on the (100) substrate and perpendicular to the [2n-n]-directions on the (n11)B substrates) in order to tailor diffusion perpendicular to the [011]-direction. By adjusting the index of the substrate the separation $S$ between steps [17] can be varied systematically from $S=0.60 \mathrm{~nm}$ for (311)B to $S=1.80 \mathrm{~nm}$ for (911)B creating the opportunity to adjust the surface diffusion pattern for adatom migration. As a result, we are able to tune the lateral alignment by manipulating surface diffusion.

This behavior can be understood since variation of the separation between surface steps affects the directional surface diffusion of the adatoms and consequently the strain profile transferred from one QD layer to the next. For example, for growth on a (100) substrate, diffusion is large along the [0-11]-direction and comparatively low along the [011]-direction. Consequently strain is much more relaxed along the [0-11]-direction than the [011]-direction.
Therefore, the strain pattern transferred from one QD layer to the next is elliptical in profile forcing the nearest neighbor QD to be along the [0-11]-direction and forming a 3D-array of QD chains. Likewise, by using a set of high index substrates the diffusion along the [0-11]-direction can be gradually tuned to be comparable to that along the [011]direction as is apparently the case for substrates ranging from (911)B (Fig. 1b) to (511)B (Fig.1c) to (311)B. For example, for (511)B the strain is most relaxed along a direction making an in-plane deflection angle $\theta$ of about 40 degrees to the [25-5]-direction forcing the nearest neighbor QD to be along the same direction. Consequently, by the vertical transfer of a corresponding strain pattern, we are able to form a 3D cubic lattice of QDs. In fact, rather remarkably, the angle $\theta$ is experimentally seen to vary linearly with $1 / \mathrm{S}$ where $S$ is the step separation [17] (Fig.2). The angle $\theta$ was evaluated by two-dimensional Fourier transforms of the AFM images (shown in Fig. 1a,b,c), and by grazingincidence $\mathrm{X}$-ray small angle scattering (GISAXS), as depicted in Fig. 1(d). The well pronounced intensity pattern of x-ray satellites peaks (Fig. 1(d)) enables a very accurate (less than one degree) analysis of the nearest-neighbor directions and the dot-dot distances while the AFM images give visual support to these measurements. Therefore, the natural surface steps on high index templates can clearly be used to tune the 2D QD lateral ordering on the surface. This is a new and useful phenomenon for engineering QD patterns with predictable ordering.

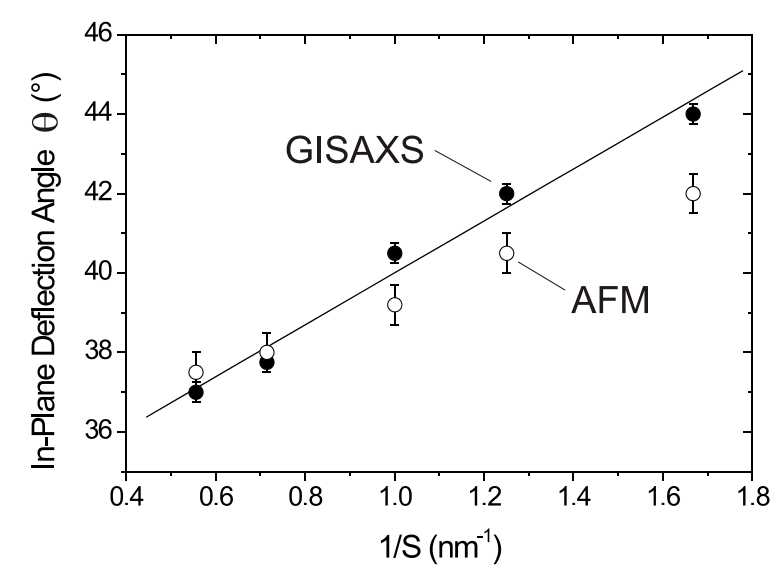

FIG. 2. In-plane deflection angle $\theta$ of the nearest-neighbor direction from $[2 n-n]$ as a function of $1 / S$ (S: nominal surface step separation) of the high index surfaces under study; +, AFM-FFT; , , GISAXS; the solid line represents a linear fit to the GISAXS data.

While the lateral ordering in our 3D QD lattices can be understood to depend on the starting substrate it is perhaps surprising that the vertical ordering is also dependent but for a totally different reason. Our observations show that the vertical ordering in $3 \mathrm{D}$ lattices is not strictly along the growth direction as might be naively expected. Rather, the vertical alignment deviates from the surface normal by a significant inclination angle $\alpha$.

This is demonstrated in Fig. 3 which shows two 2D sections of the $\mathrm{x}$-ray diffuse scattered intensity from the (511) B-sample in the vicinity of the GaAs 5-11 reciprocal 
lattice point. Fig. 3(a) gives the x-ray diffuse scattering along the [25-5]-direction while Fig. 3(b) gives the $\mathrm{x}$-ray diffuse scattering along the [011]-direction. Beside the modulation of the crystal truncation rod (located at $\mathrm{q}_{25-5}=0$ and $\mathrm{q}_{011}=$ 0 ), which is caused by the mean superlattice period $\left(\mathrm{t}_{\mathrm{SL}}=32.5 \mathrm{~nm} \pm 0.1 \mathrm{~nm}\right)$ along the growth direction, considerable $\mathrm{x}$-ray diffuse scattering is observed and is mainly created by local strain fluctuations inside the sample. The diffuse intensity is vertically bunched into so-called resonant diffuse scattering (RDS) sheets, indicating that the lateral QD positions are vertically correlated. Horizontally, within the RDS sheets, pronounced intensity maxima occur, which are caused by lateral ordering of the QDs. In the direction along [25-5] [Fig. 3(a)] the RDS sheets are tilted from the exact horizontal orientation, and the angle of inclination $\alpha$ exactly corresponds to the deviation of the vertical arrangement of the QD positions from the surface normal towards the [25-5]-direction [18]. By contrast, the inclination vanishes in the direction along [011] [Fig. 3(b)]. The inclination angles $\alpha$ were determined experimentally for all samples under study [Fig. 4(b)], with the important result that the inclination always points towards the [2n-n]direction, i.e. the direction perpendicular to the nominal step edge direction. On the other hand the inclination towards [011] always vanishes.

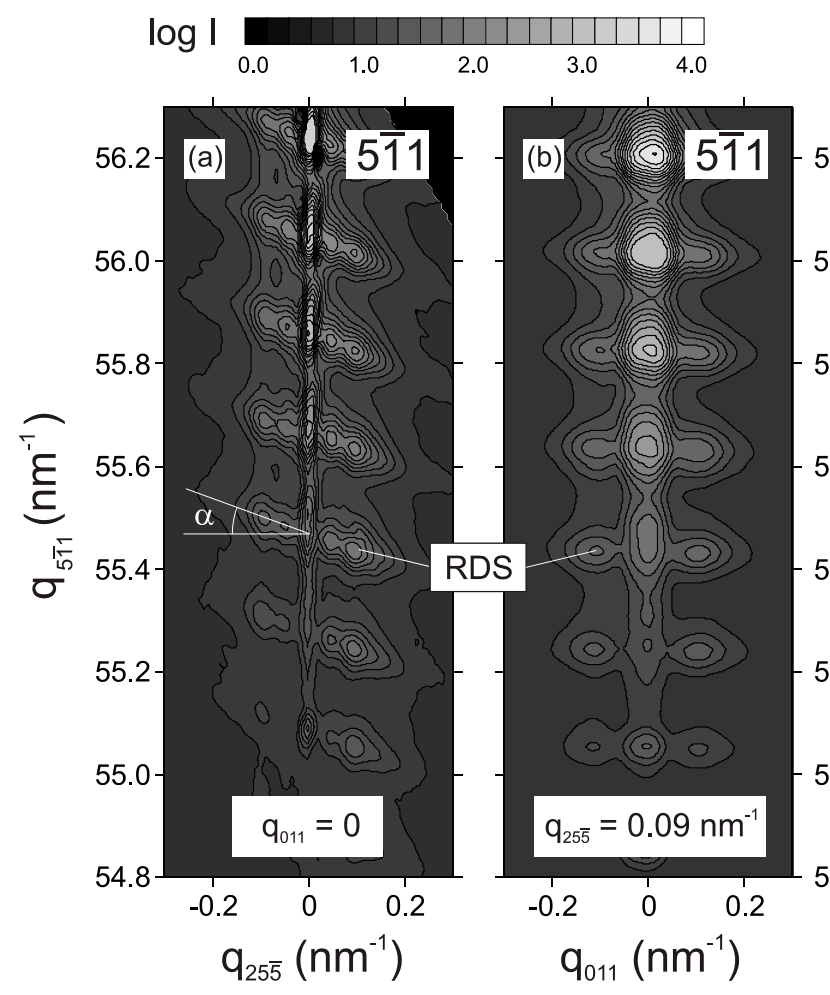

FIG.3. X-ray diffuse scattering from the (511)B-sample in the vicinity of the GaAs 5-11 reciprocal lattice point. In the direction (a) along [25-5] the x-ray diffuse scattering (which is concentrated in RDS-sheets) is inclined by the angle $\alpha$ while in (b) the direction along [011] the RDS-sheets are oriented horizontally. This proves inclined vertical inheritance of the horizontal QD positions towards the [25-5]-direction, i.e., perpendicular to the surface step edge direction.
In order to understand this behavior, numerical model calculations using elasticity theory were performed. Holý et al. [15] treated the QDs as point sources thus neglecting the actual shape and size of the QDs that are embedded in an elastic matrix. With that approximation only the elastic far field around the QDs can be appropriately described. The far field behavior solely depends on the elastic properties of the surrounding material and is thus an intrinsic material property. For the samples under study here, the horizontal extension of the QDs is of the same magnitude as the thickness of the spacer layer $(\mathrm{t}=30 \mathrm{~nm})$. Therefore, results of exact theoretical treatment are expected to still depend on the actual shape and size of the QDs. Our calculations are based on the finite-element method (FEM) and take into account the QD size, shape and the full elastic anisotropy of the involved materials. It is, however, noteworthy that, owing to the comparatively low stress at the surface the exact atomistic two-fold symmetry of the material as discussed by Pryor et al. [19] need not be considered. Results of the elastic energy density performed for a lens shaped $\mathrm{In}_{0.40} \mathrm{Ga}_{0.60} \mathrm{As}$ dot (base width $\mathrm{w}=30 \mathrm{~nm}$, height $\mathrm{h}=5 \mathrm{~nm}$ ) are presented in Fig. 4(a). The dot is located $30 \mathrm{~nm}$ below the (511) B surface of a GaAs matrix. A clear shift of the minimum position towards the [25-5]-direction is observed, confirming the experimental result of inclined inheritance towards this direction [Fig. 3(a)].

A series of FEM calculations was performed for different GaAs surface orientations of the type $(n 11) B$, where $n$ is equal to 9, 7, 5, 4, and 3. Angles $\alpha$ of inclined inheritance are extracted from these calculations and are compared with 56.2 corresponding experimental results [Fig. 4(b)]. For (111)B and (100) surface orientations the energy density shows pronounced minima just above the underlying QD in the previous layer, thus favoring exact vertical correlation [15]. However, for surface orientations on the path between (100) 55.8 and (111)B, i.e. (911)B, (711)B, ..., (311)B, remarkably high values for $\alpha$ are observed, with a maximum for the (711)B surface orientation exactly matching the experimental observation. We should emphasize that even a rather small deviation from (100) leads to a remarkably inclined 55.4 inheritance [see calculated data point for $(1511) \mathrm{B}$ in Fig. 4(b)].

Overall agreement between the FEM calculations and the 55.2 experimental values for $\alpha$ is observed, however, the experimentally derived values are slightly larger than the 55.0 predicted ones. This deviation cannot be explained by the finite size of the QDs. Our calculations show that increased QD sizes - as compared to the spacer thickness - lead to a reduction of the inclination angle $\alpha$, while a decrease of the QD size does not further significantly affect our results. Obviously, the present geometrical parameters $(\mathrm{w}=30 \mathrm{~nm}, \mathrm{~h}$ $=5 \mathrm{~nm}, \mathrm{t}=30 \mathrm{~nm}$ ) are close to the 'far-field limit' considered by Holý et al [15] for which the inclined inheritance angle is insensitive to the finite QD size. This can be also inspected by comparing $\alpha_{311}=10^{\circ}$ as predicted by Holý et al. [15] with $\alpha_{311}=11^{\circ}$ as calculated in our study [20].

In conclusion, we have observed and explained the formation of 3D ordered QD lattices when the substrate orientation is varied from (100) to (311) B in the direction 
towards (111)B. B-type steps oriented along the [011]direction, were used to fine-tune the adatom surface diffusion in order to form cubic lattices. This leads to a deviation of the QD nearest-neighbor direction that can be described by an in-plane deflection angle $\theta$ measured relative to the [2nn]-direction, i.e., perpendicular to the B-type step edge direction. A linear relationship between 1/S (S: nominal Btype separation between steps) and the deflection angle $\theta$ was experimentally observed. Meanwhile, these changes are coupled to changes in the alignment along the growth direction. Theoretical investigations based on a linear elasticity theory show a systematic variation of the angle $\alpha$ of vertical inheritance of the lateral QD positions when the surface orientation is changed from (100) towards (111)B. The calculated values for the inclination angle $\alpha$ are mainly determined by the elastic anisotropy of the GaAs matrix and are in excellent agreement with experimental findings. Thus, we have demonstrated that the diffusion characteristics of the surface and the elastic anisotropy of the matrix, provides an excellent opportunity to self-organize 3D QD lattices with predictable lateral and vertical ordering.
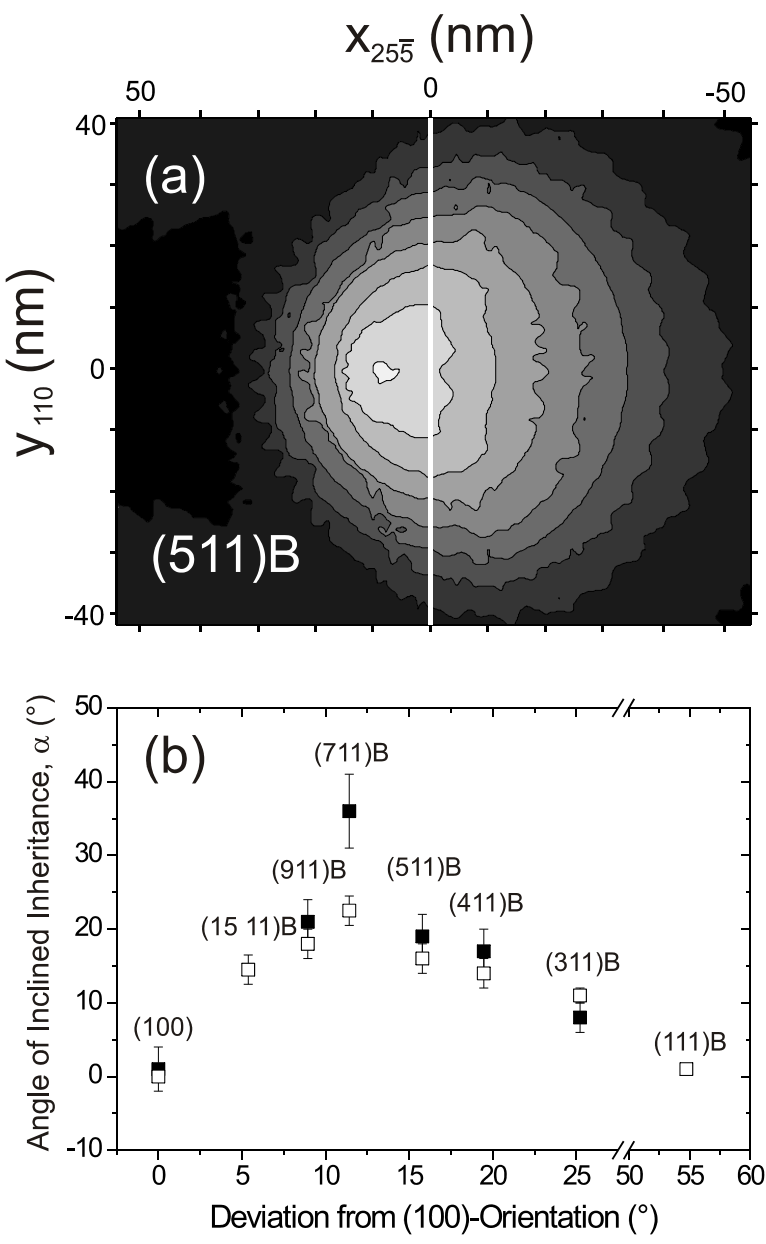

FIG. 4. (a) Calculated elastic strain energy density (linear gray scale in arb. units) on the (511)B-surface above a strained $\mathrm{In}_{0.40} \mathrm{Ga}_{0.60} \mathrm{As}$ QD which is embedded into a GaAs matrix at $30 \mathrm{~nm}$ below the surface; (b) angle of inclined inheritance $\alpha$ of the lateral QD positions to the surface normal; ( , calculations; $*$, experimental.
We thank HASYLAB staff for technical support with the $\mathrm{X}$-ray experiments. This work was supported by Deutsche Forschungsgemeinschaft (SFB 296).

[1] D. Bimberg, M. Grundmann, and N. N. Ledentsov, Quantum Dot Heterostructures (Wiley, Chichester, NY, 1999).

[2] V. Shchukin, N. N. Ledentsov, and D. Bimberg, Epitaxy of Nanostructures, Nanoscience and Technology (Springer, Berlin, 2003).

[3] F. Leroy et al., Appl. Phys. Lett. 80, 3078 (2002).

[4] T.I.Kamins and R.S. Williams, Appl. Phys. Lett. 71, 1201 (1997).

[5] R. Songmuang et al., Appl. Phys. Lett. 82, 2892 (2003)

[6] H. Lee et al., Appl. Phys. Lett. 78, 105 (2001).

[7] D. Leonard et al., Appl. Phys. Lett. 63, 3203 (1993).

[8] T. Mano et al., Appl. Phys. Lett. 81, 1705 (2002).

[9] Yu. I. Mazur et al., Appl. Phys. Lett. 83, 987 (2003).

[10] G. Springholz et al., Science 282, 734 (1998).

[11] G. Springholz et al., Phys. Rev. Lett. 84, 4669 (2000).

[12] Zh. M. Wang et al., Appl. Phys. Lett. 84, 1931 (2004).

[13] J. Tersoff, C. Teichert, and M.G. Lagally, Phys. Rev. Lett. 76, 1675 (1996).

[14] Q. Xie et al., Phys. Rev. Lett. 75, 2542 (1995).

[15] V. Holý et al., Phys. Rev. Lett. 83, 356 (1999).

[16] M. Schmidbauer, X-Ray Diffuse Scattering from SelfOrganized Mesoscopic Semiconductor Structures (Springer, Berlin, 2004).

[17] The nominal step separation $\mathrm{S}$ of a GaAs (n11)-surface consisting of (001) terraces is given as $\mathrm{S}=\frac{0.5653 \mathrm{~nm}}{2} \frac{\mathrm{n}}{\sqrt{2}}=\mathrm{n} \times 0.20 \mathrm{~nm}$

[18] E.A. Kondrashkina et al., Phys. Rev. B 56, 10469 (1997).

[19] C. Pryor et al., J. Appl. Phys. 83, 2548 (1998).

[20] Within the framework of linear continuum elasticity theory the crystallographic orientations (311)A and (311)B of GaAs are equivalent. 